Original Research Paper

\title{
Arduino Based IOT Platform for Remote Monitoring of Heart Attacks and Patients Falls
}

\author{
Manikandan Shanmugam and Monisha Singh \\ Department of Computer Science, Christ University, Bangalore, India
}

\author{
Article history \\ Received: 02-02-2018 \\ Revised: 12-03-2018 \\ Accepted: 28-04-2018 \\ Corresponding Author: \\ Manikandan Shanmugam \\ Department of Computer \\ Science, Christ University, \\ Bangalore, India \\ Email: \\ manikandan.s@mca.christuniv \\ ersity.in
}

\begin{abstract}
Internet of things (IoT) is a networking concept that allows connection of various smart devices. This concept plays a huge role in the healthcare industry. The developed system is a working prototype for realtime monitoring of patient falls and heart attacks. The process of developing this system included an architecture, which was built using Arduino UNO and Arduino NANO along with pulse sensors and accelerometer sensors. The main idea is to collect health-related data from time to time and the collected data is made available using a real-time interface called Thingspeak. With the help of this process, the person can be monitored from time to time without any hassle. The proposed system also makes use of delivering notifications at the time of emergency using the GSM technology, which is embedded with the Arduino architecture. This system will be of greater help to elderly people, people suffering from Frankenstein disease or people who are in a history of getting heart attacks due to genetic disorders.
\end{abstract}

Keywords: Arduino, IoT, GSM, Thingspeak, Sensors, Healthcare

\section{Introduction}

Internet of Things (IoT) is one of the emerging IT technology changing the way of connectivity across devices and removes the barriers between devices and humans by providing a virtual communication between them. The cost of Broadband internet is effectively reduced when compared to the previous scenarios and the number of people and devices getting connected every day keeps increasing enormously, so a virtual Eco space technology was introduced in order to provide various operations such as data collection and visualization across devices which are connected and making them available to the end user by which the users can make collective decisions (Muhammad et al., 2017; Abdelgawad and Yelamarthi, 2016).

Arduino is an architectural board used for electronic coding devices. The entire family of these microcontrollers is of ATMEGA sheets. The architecture part consists of 14 digital input and output pins, power out, reset button and a USB connection. Within this architecture, the sensors are connected which in turn collects the user's health-related data from time to time and makes it available to the user. The various sensors which are used is accelerometer and pulse rate. The basic functionality of accelerometer sensor is to detect person movements and the pulse rate sensor is used detect the person's heart beat over a minute, certain predefined value will be coded within the Arduino IDE and a condition will be specified, if so there is an overshoot or underrate of predefined values a resulting notifications statement can be specified within the IDE.

The accelerometer sensor's power consumption is relatively less and is directly connected to the Arduino board. There is a various number of series in accelerometer sensor such as ADXL320-330 and the collected acceleration data will be made available in the serial monitor within the IDE (Viet and Choi, 2012).

The pulse sensor will be connected directly towards the input signal unit of the board, which helps in detecting the user's live heart rate. The sensor keeps blinking a green signal on which the user's finger edge will be placed, the reflecting wavelength signal reads the user's heart rate and makes it available in the serial monitor.

The pulse sensor is a small chip will be placed and tapped around the user's index finger whereas the accelerometer sensor will be placed around the user's thigh portion. Since the user remains seated in a wheelchair, he remains in a stagnant position, so any tilt 
from the stagnant position will trigger the sensor, as there is a change in the predefined value. At last, the main circuit board is attached below the user's wheelchair. The developed system is prototype model to detect the heart attacks and save one's life prior the danger, provided with a better budget, this model can be made more small and compact.

Thingspeak is one of the widely spoken IoT analytics technology, which is used for the visualization purpose of the collected data. It does the basic task of collection, analyze and providing actions based on the derived results. The Thingspeak API will be embedded along the Arduino IDE by making use of an API key provided to a particular user after login. This API key provides a connection between the IDE and the online Thingspeak tool, so the data collected from sensors will be analyzed and later a pictorial graph will be provided to the user to make decisions based on the graph. There are different types of views provided within the tool such as private and public, based the user requires the setting can be changed. By making the data public, multiple users can view and access the data whereas in private view only the authenticated users will be provided access to the user's data. There is also an opportunity to share the results with multiple users using this online IoT analytics tool (Li et al., 2016; Muhammad et al., 2017; Satija et al., 2017).

To provide a summarized view, the system performs its functionality using the Arduino architecture by collecting user related pulse rate and acceleration data using appropriate sensors and makes it available in Thingspeak for further processing; the system also embeds a GSM module to provide immediate notification at the time of emergency. The basic idea is to provide remote monitoring of heart attacks and patient falls by restricting it to a particular user.

\section{Literature Review}

\section{A. Arduino based Fall Detection and Tracking of Elderly}

This paper mainly focuses on the health risks associated with elderly people and provides a prototype model to detect the accidental falls. Since the cost associated with the caretakers are ever increasing, the present system will locate the position of the patient when the fall has occurred and intimates the peer group using the GSM technology. The system makes use of sensors such as MPU-6050 and GY-521. The system provides a solution for location tracking and detecting patient falls. The main idea behind this system was to reduce the working cost of a caretaker and provides a minimalized effort system for monitoring using Arduino (Li et al., 2016).

\section{B. Real Time Health Monitoring System using Arduino}

This paper also provides a prototype model, which is used to monitor the various aspects of a human body such as breathing oxygen level, temperature level and the live heart rate level by making use of appropriate biosensors. Most of the present system collects the user data using wires, which made the system clumsy and inefficient, so the proposed system makes of wireless [WiFi] technology to collect the health-related data. All of the collected data will be stored and processed in the centralized database if there is any mismatch in the prescribed values a notification will be sent to networked medical associates. All the data, which are collected from sensors, are connected to Arduino microcontroller and results are maintained in the database. The further analysis of the simulated results is done using Proteus software (Li et al., 2016; Ahn et al., 2013).

\section{Application of Arduino Based Platform for Wearable Health Monitoring System}

In this study, the health-related data is collected by embedding the sensors within a shirt, since the aging population is increasing day by day due to advanced medication and the number of diseases is increasing at an alarming rate, a constant check over one's body is always necessary. The proposed system provides an ambulatory device, which constantly checks various aspects such as dermal activity, temperature and heart rate. By combining the results of all these aspects, a clear view can be derived concerning a patient's body. The heart attack condition is also taken into consideration while implementing this system. The main idea of this paper is to collect the data related to personal health by both increasing the number of sensors in a shirt which by default will increase the efficiency of the proposed system. This system will be useful to military patients, people suffering from chronic diseases, athletes and elderly personnel's. This system made use of Arduino version 1.3 to simulate the results; the core Arduino files were rewritten to modify the behaviour of the AVR chip. The system also provides an opportunity to add or remove sensors within the shirts based on the patient's requirement (Muhammad et al., 2017; Viet and Choi, 2012).

\section{Wireless patch sensor for Remote Monitoring of Heart rate, Respiration, Activity and falls}

In this study, the major activities that the paper discusses are about the remote monitoring of health activities, at-home screening and providing notifications to the user at the time of serious situations such as heart attacks and respiratory problems. This paper consists of proven results of Bluetooth Low Energy sensors, which 
in turn contains two ECG sensors and an accelerometer along with a transceiver. The proposed system was tested among a group of adults, which monitors their heart rate, breathing rate and compares the variability between them to derive accurate results. The breathing process was natural evaluated by making the individuals perform various activities such as walking and cycling. When a combination of ECG and accelerometer is provided better results can be derived based on the respiratory rate. A wireless patch sensor was proposed in the system which monitors some important aspects of the human body later the derived results are compared with the results derived from the traditional system and better decisions are made (Abdelgawad and Yelamarthi, 2016; Chan et al., 2013).

\section{E. Fall Detection System for Elderly Person Monitoring Using GSM Network}

This paper monitors the fall rate related to the elderly age group. According to WHO major cause for severe injuries are caused due to patient falls. When a patient is being injured due to a fall, it may affect his/her day-to-day activities and bring a change in the routine. The major sensors used for fall detection under this system was ultrasonic and accelerometer sensors, the reason to choose these sensors where that it can is both cost effective and provides better results. A constant value is predefined and if there is an overshoot of values, it might end up transmitting a notification is RF transmitter and receiver. When there is an emergency, a notification will be sent to the peer group using the GSM technology. The major operations performed in this system is that the location of the user is determined later using the accelerometer sensors the patient moves can be judged and the fall can be detected using the ultrasonic sensors (Shuo, 2015; Muhammad et al., 2017).

\section{F. WeCare: An Intelligent Badge for Elderly Danger Detection and Alert.}

The proposed is named as WeCare, were the basic idea is to monitor elderly people and chronic disease patients who require constant monitoring over the health. An accelerometer sensor is attached to the system, which monitors the patient falls, patient movements as well the regular activities of the patient. A service center website was developed to intimate the user in case of emergency. Then the system was evaluated to measure the performance and the system provided efficient results as expected. They monitored activities of the user such as stair climbing and regular home events. The developers of this WeCare system are currently working on improving their present system and thereby improving their accuracy (Shuo, 2015.

\section{G. Fall Detection System Using Arduino Fio}

This paper also considered the elderly population is belonging to a nuclear family they require a caretaker to have a constant check on their health, but the system varies from others because this system was developed using a new version of Arduino called as Fio. By making use of Fio, the developers were able to derive. Initially, the system began by setting threshold values, tests were conducted on the accuracy and sensitivity of the system and the previously set benchmark value was reached. The future work of the system is to improve the benchmark value by reducing the false positive rate. This can be done by incorporating multiple sensors such as infrared sensors and cameras so that the actual benchmark value can be increased. The system also aims at improving the email reply system by making the caretakers reply to the emails sent at the time of emergency by constantly sending them emails until a reply is received from the caretakers (Viet and Choi, 2012; Shuo, 2015).

\section{H. Structural Health Monitoring: Internet of Things}

This paper focuses on the various capabilities that can be achieved when IoT can be combined with the regular health monitoring system. The proposed SHM system monitors the complete structural health by combining the SHM with IoT. The main module makes use of Raspberry $\mathrm{Pi}$ along with $\mathrm{WiFi}$ and NRF transceiver module. Multiple piezoelectric sensors are integrated within the module so that the data is collected smoothly. The pro-trinket sensor is embedded within the system to make sure of the damage occurred and the position in which it occurred.

\section{Smart Health Solution Integrating IoT and Cloud: A Case Study of Voice Pathology Monitoring}

The framework mainly focuses on integrating Cloud computing and IoT technologies to provide better solutions concerning the healthcare industry. Since these two technologies play a vital role in various applications regarding scalability, security, accessibility and flexibility adopting these technologies alongside with latest smartphones can bring a change in the present state of the healthcare industry. This paper mainly focuses on bringing an improvement in the voice pathology sector of healthcare, the detection of voice wavelengths is done using binary patterns and a machine learning classifier called ELM was proposed to detect the changes occurred in the voice wavelengths and the accuracy achieved is comparatively more when compared to the present system. The aim of this system is to develop to improve the user experience and making the data available without any hassle, the security part of the system is taken care by the service provider. The future work of the system relies on improving the scalability and 
dynamic collection of voice inputs efficiently (Abdelgawad and Yelamarthi, 2016; Chan et al., 2013).

\section{J. Fall Detection based on Movement and Smart Phone Technology}

In this study, the main idea is to detect the patient falls but using the smartphone technology, this system monitors the day-to-day activities of the user and suggests useful advice to the peer healthcare applications installed on the user phone. As falling event can cause a critical pain regarding elderly people but determining such situations before happening seems to be a difficult task to carry research. Such accidents can similarly lead to a situation of the patient getting heart attacks or getting respiratory problems. Even though there is rise in the number of sensors making use of them in wearable devices and getting accurate results still seems to be a problematic area, as the person needs to be equipped with all these sensors in a daily basis, so the proposed system came up with the idea of collecting these data in order to predict patient falls using the mobile devices as smartphones play an impeccable part in our day to day lives. By using the acceleration and orientation data from the mobile devices a decent level of predicting patient fall can be derived (Rajalakhshmi and Nikilla, 2016; Chan et al., 2013).

\section{K. Design and Implementation of an Accidental Fall Detection System for Elderly}

This paper also concentrates on the elderly age group, which is considered to be a global problem. When considering such population, an all-time surveillance system is necessary to monitor such age group. This system also portrays the idea of helping the elderly population at the time of fall by notifying the user using public mobile services. The major components of this system are microcontroller to which the acceleration sensors are connected and the user is notified using the GSM technology, the number to which the message needs to be sent will be set as a constant value while coding the microcontroller, normally the patient's family member numbers will be coded as a default constant value. The functional working of all the components is later discussed in this study (Muhammad et al., 2017).

\section{Survey on Fall Detection and Fall Prevention Using Wearable and External Sensors}

In this study, a survey was made on the number of users who are treated due to fall-related injuries and numbers crossed over 1.6 million of adults over the years, since this seems to be of greater threat a mechanism was needed to bring in control over this alarming rate of patient falls. This paper provides a comparative view of the various mechanisms, which are already implemented in the field of fall detection and fall prevention and provides a survey result by stating which technique holds in best. The different approaches followed in each system, the various sensors used and their results are discussed in this study, the risk factors associated with these techniques are also discussed (Muhammad et al., 2017; Satija et al., 2017).

\section{Real-Time Signal Quality-Aware ECG Telemetry System for IoT-Based Healthcare Monitroing}

In this study, an IoT based ECG system was developed to monitor one's health. The present system consists of signal assessment module to improve the signal quality, ECG sensors and transmission module to send out the received data. The main idea of the system is to improve the signal transmission quality of ECG telemetry by incorporating a lightweight design and development methods. Once the results are derived, it is classified into an acceptable class and non-acceptable class. The framework is built using a smartphone, Arduino, ECG data collecting sensors and Bluetooth for data results transmission. The framework is also tested against Physio net database and MIT-BIH arrhythmia for validation purpose. The results proved that the proposed system performs better than the existing framework. This method can be incorporated in healthcare agencies using traditional ECG data collection methods (Chan et al., 2013; Shuo, 2015).

\section{Framework}

In this section a detailed diagrammatic representation is provided regarding the proposed system architecture. The actual workflow begins with the components getting connected towards the Arduino Board that is the accelerometer sensor which the person movements and the Pulse rate sensor which detects the person heartbeats will be connected to the board. In addition to this, the architecture also combines the feature of providing notifications at the of emergency, so there is the GSM module which in turn will be connected towards the board (Abdelgawad and Yelamarthi, 2016).

In the below diagrams (Fig. 1 and 2), a connection methodology of the GSM module is shown. The system shows that there are an Arduino board and GSM sim slot available to add the sim number to which the message needs to be sent along with the ground and power supply pins are connected towards the board.

To provide a summarized view of the architecture the four major components such as accelerometer sensor, pulse rate sensor, Arduino and GSM modules are connected and interrelated for it to exchange information with each other (Rajalakhshmi and Nikilla, 2016). 


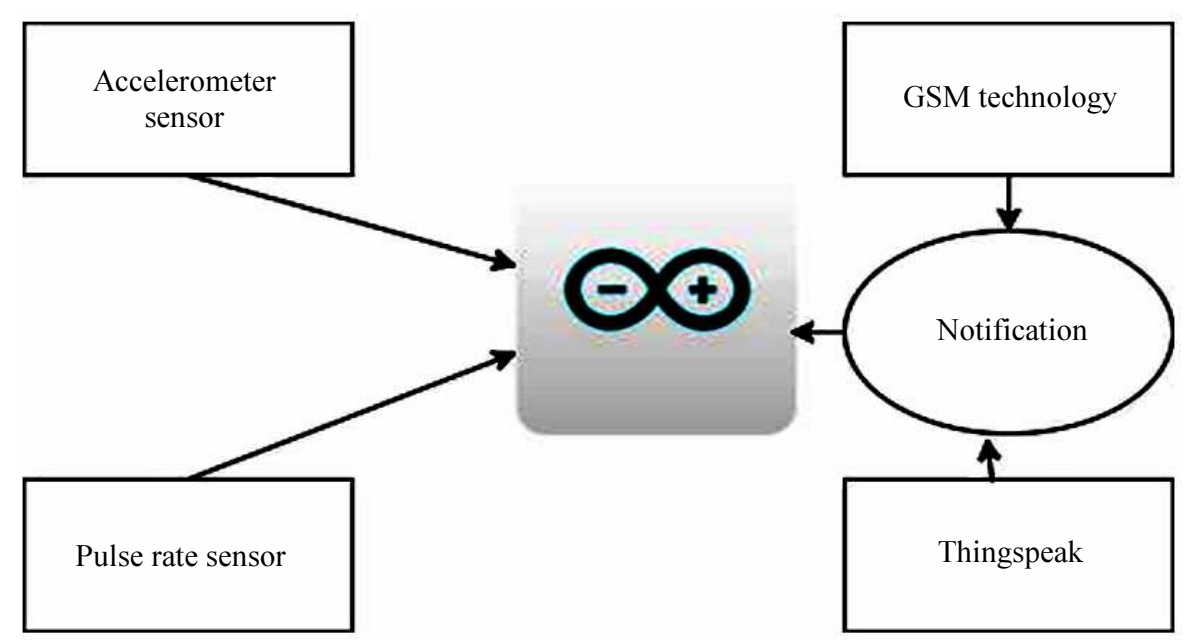

Fig. 1: Architectural framework of the developed system

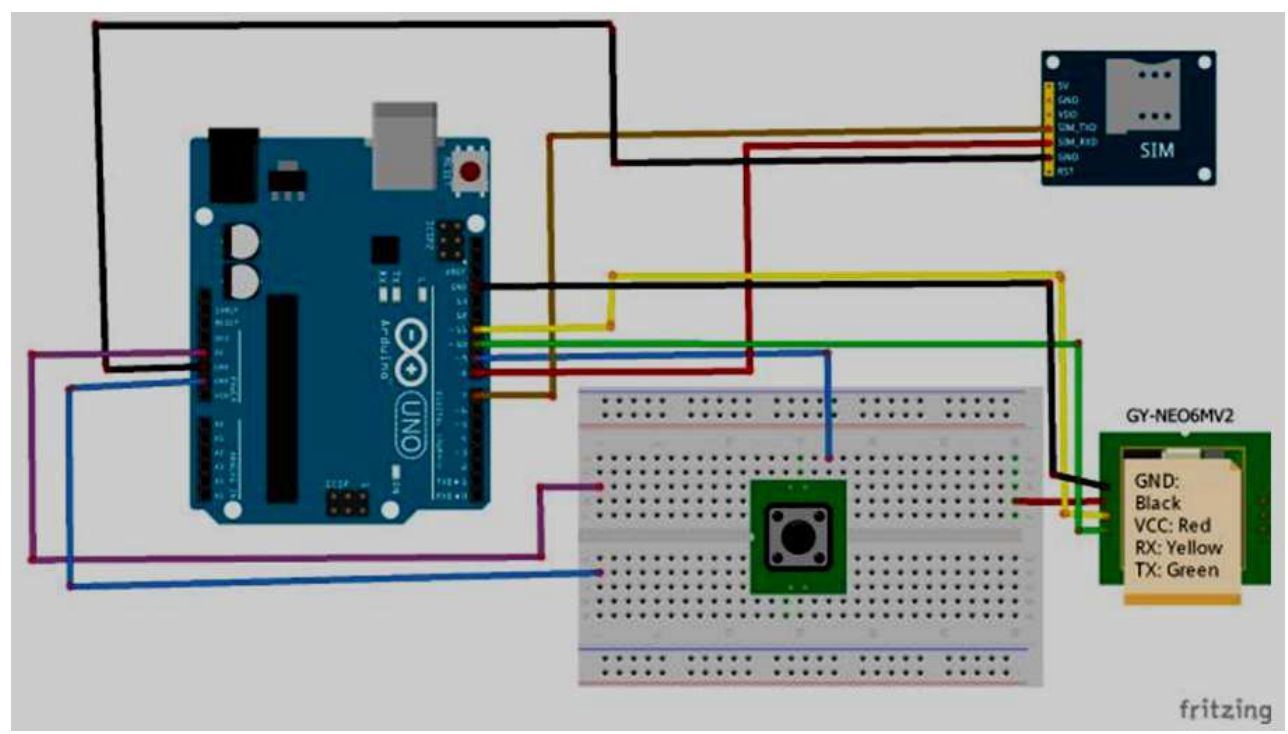

Fig. 2: GSM module

\section{Implementation}

The developed prototype model consists of the following functionalities. This section majorly concentrates on the connectivity methods followed and summarized the overall way of the proposed system implementation.

\section{A. Arduino UNO and Arduino Nano}

This Arduino board serves as a centralized architecture were the various working components such as pulse rate sensor and accelerometer sensors are connected. This board serves to be a hub for various system components used in this system. The various input and output pins along with reset and ground pins serve their purpose as discussed in the earlier phase of the paper.

\section{B. Pulse Rate Sensor}

The above diagram shows the way in which the input and output signals of the pulse rate sensor is connected towards the Arduino board. The sensor collects the heartbeat data and transfers it to the Arduino, which in turn can be viewed in the terminal using the serial monitor interface (Rajalakhshmi and Nikilla, 2016).

\section{Accelerometer Sensor}

The above diagram shows the way in which the input and output signals of the accelerometer sensor is connected towards the Arduino board. The sensor collects the acceleration or the person movement's data and transfers it to the Arduino, which in turn can be viewed in the terminal using the serial monitor interface. 
The Fig. 3 and 4 provides an outer view of the arduino board to get a clear idea regarding the connectivity.

\section{Thingspeak Interface}

The collected data from accelerometer sensor and pulse rate sensor will be collected through the board made available using an Online IoT analytics tool known as Thingspeak.

By using this tool, a public view option can opt so that the data can be shared and accessed by multiple users. So the patient-related data can be viewed by the peer groups or even by the doctors from anywhere and anytime.

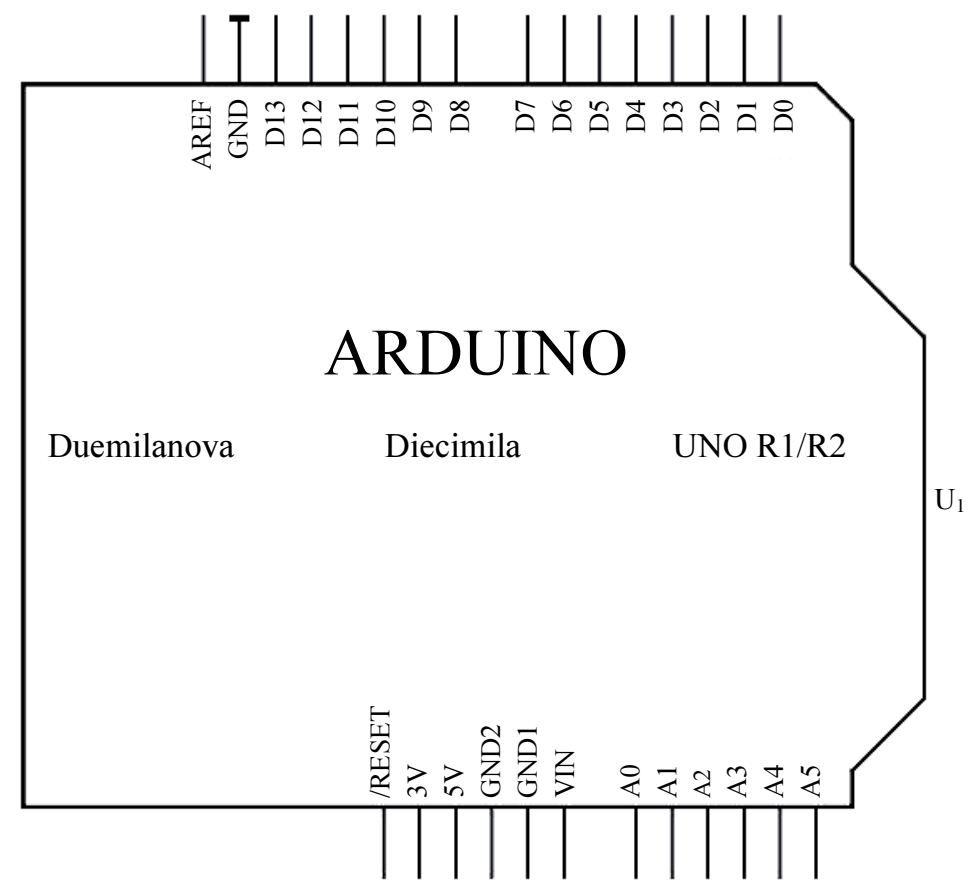

Fig. 3: Arduino circuit design

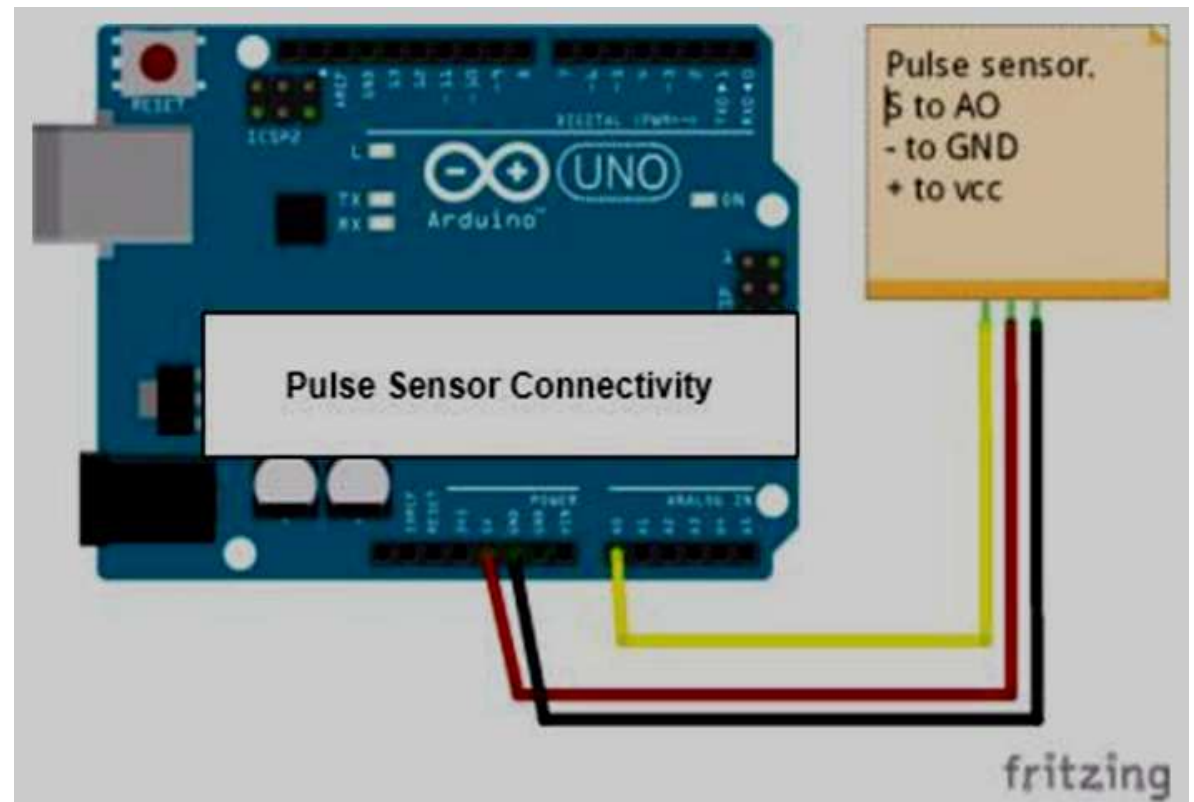

Fig. 4: Pulse sensor connectivity 
Once the data related to an individual's heartbeat is collected using the pulse sensor, the same data can be visualized within no time in the Thingspeak interface. By doing so, the individual's health advisor can monitor the person's heart beat and other related details can be analyzed based on this medicine prescription becomes an easier task. Since there is no need for the person to spend time and money to meet his/her health advisor. The Fig. 5 shows the complete circuit view of arduino once the accelerometer is connected. The Fig. 6 and 7 shows the thingspeak visulization after the data is been collected from the patient's body.

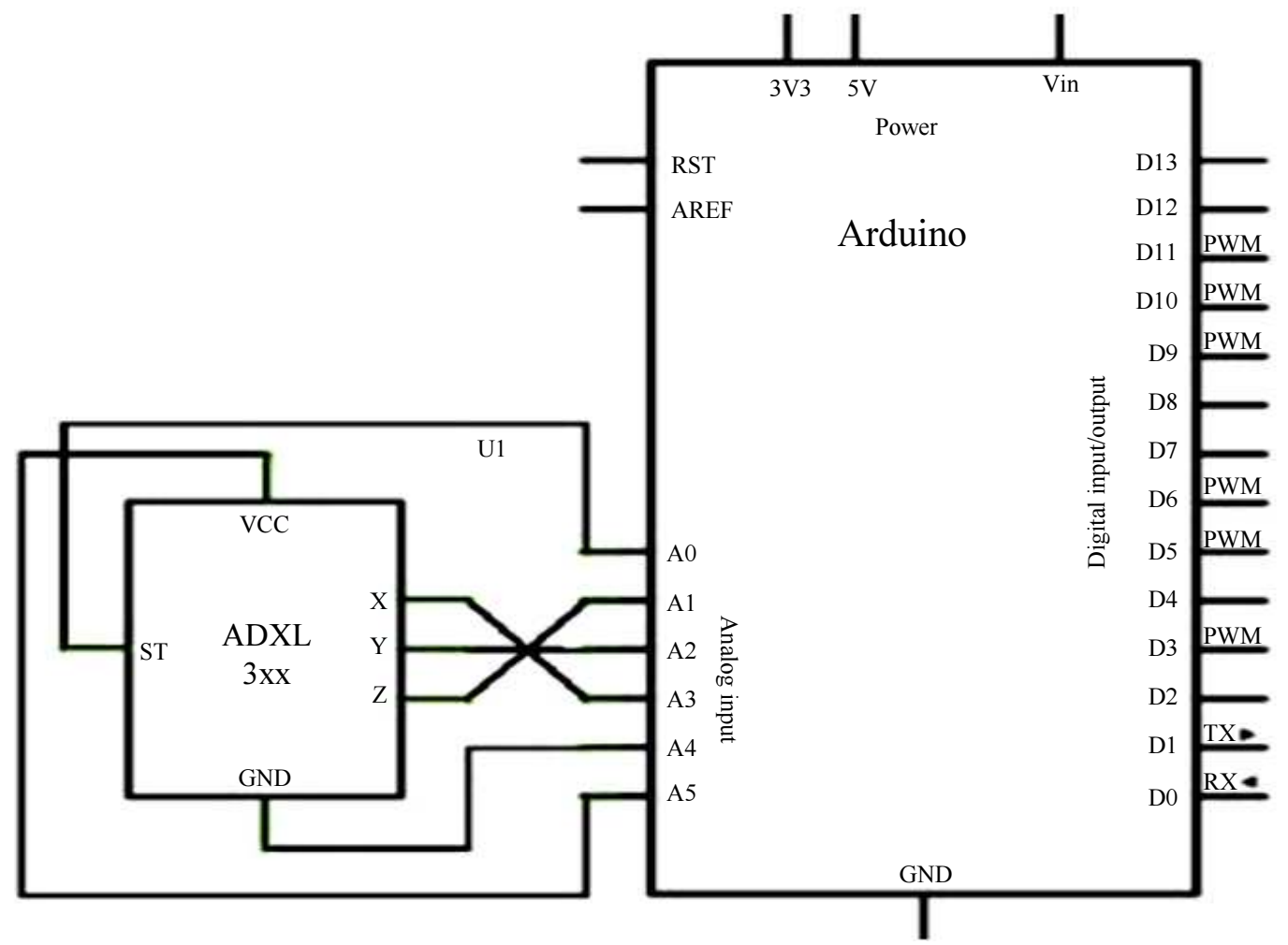

Fig. 5: Accelerometer sensor connectivity

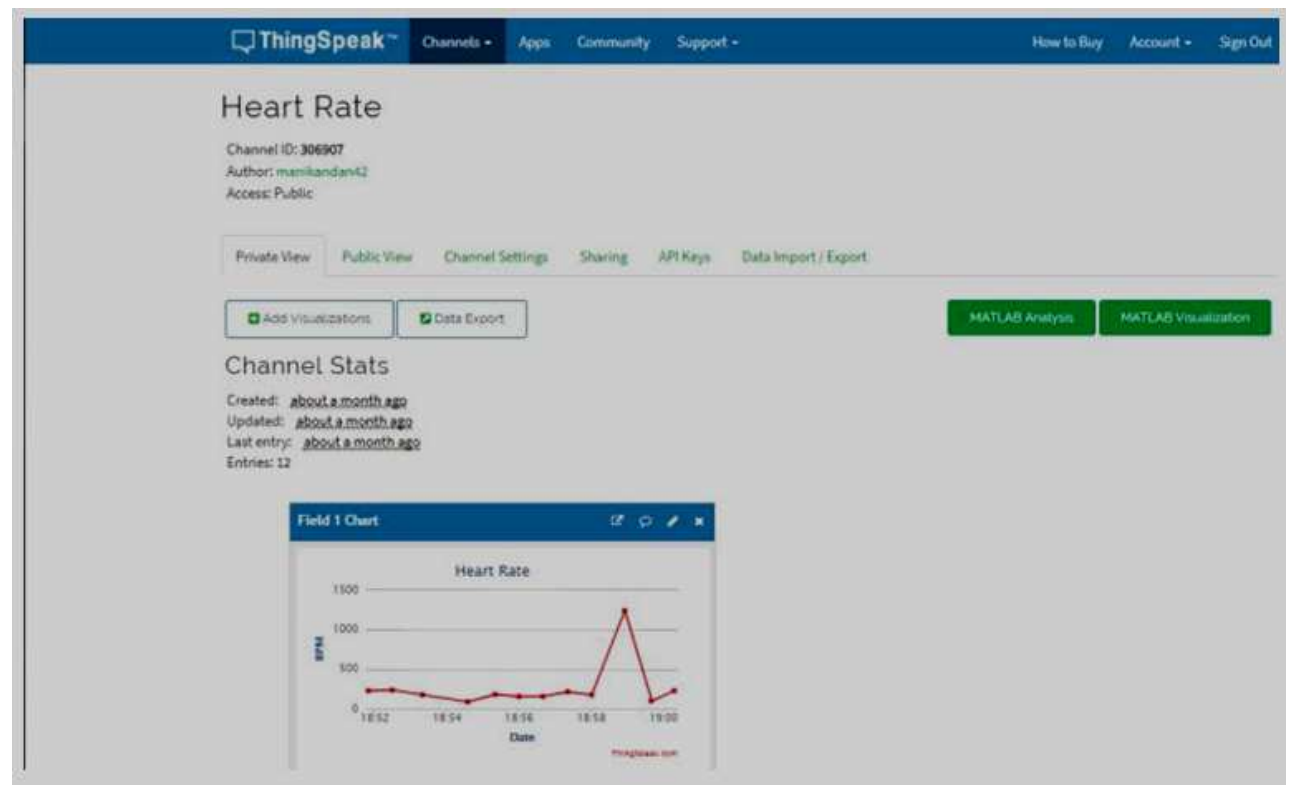

Fig. 6: Thingspeak front-end 


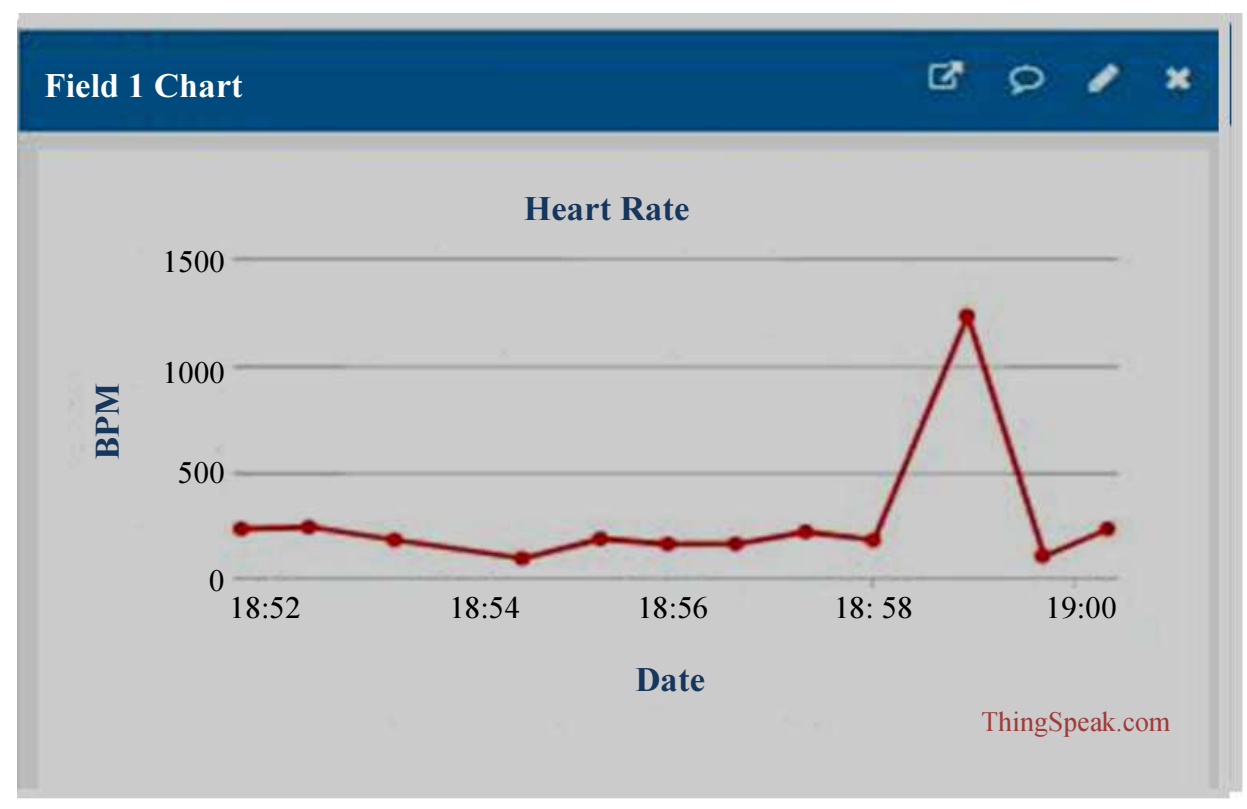

Fig. 7: Thingspeak heart beat visualization

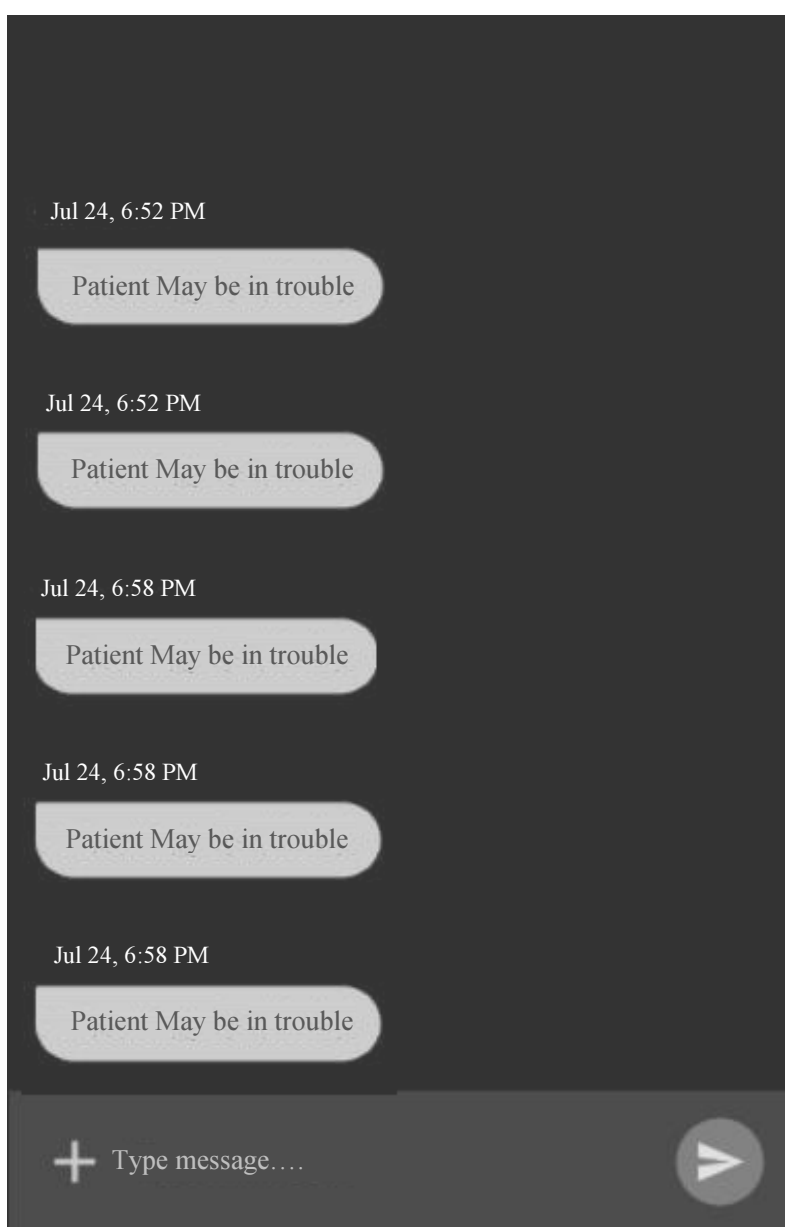

Fig. 8: Message in User's mobile stating that the patient might be in trouble

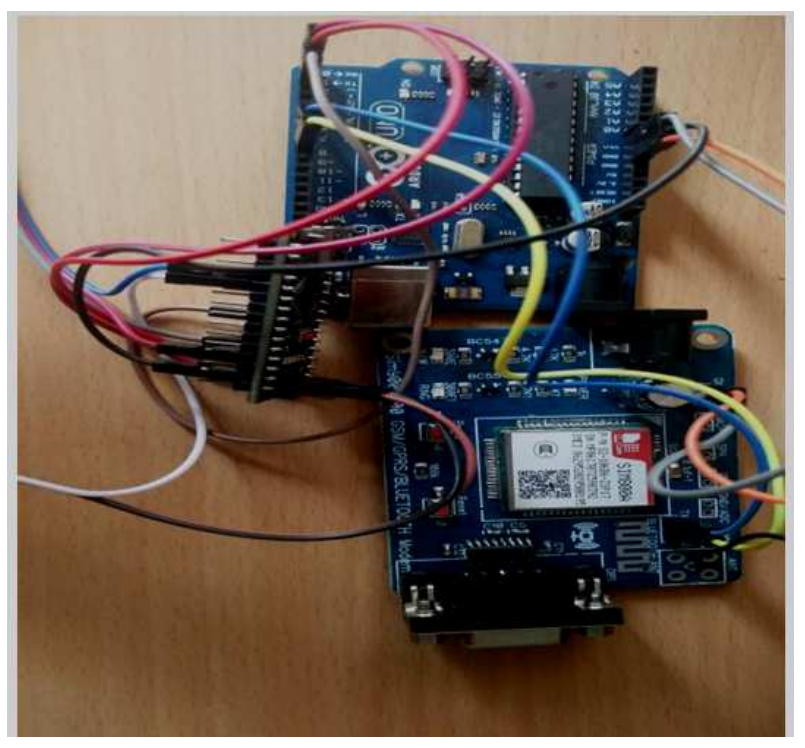

Fig. 9: Arduino board connectivity

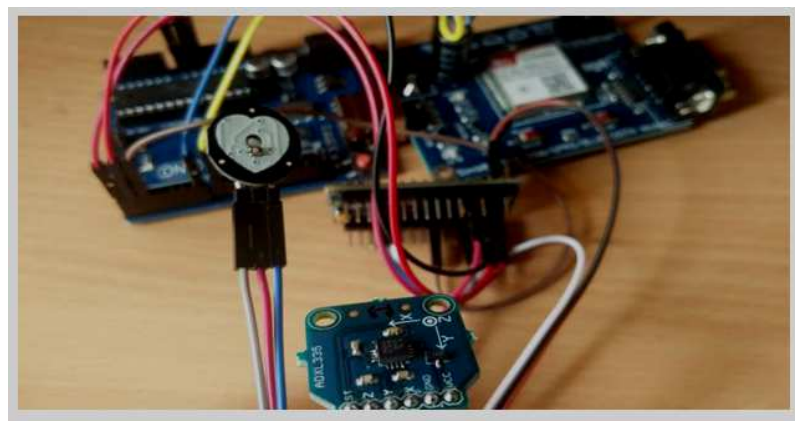

Fig. 10: Pulse rate sensor and Accelerometer sensor 


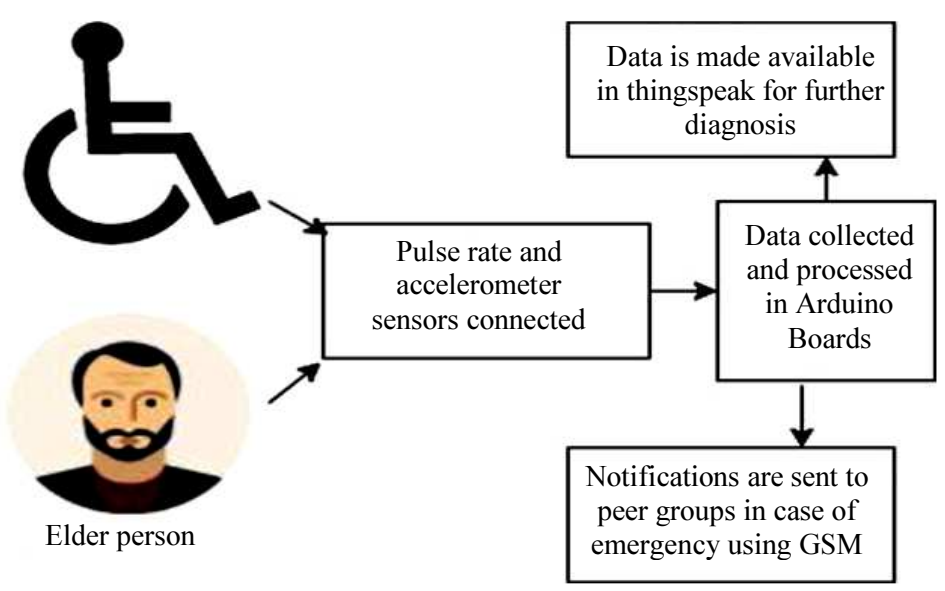

Fig. 11: A summarized view of the proposed system

\section{E. Global System for Mobile [GSM]}

We make use of this technology mainly for the notification purpose. In case of an emergency situation such as when there is an overshoot or undershoot heart rate below the prescribed level, immediately a text message will be sent out to the person's peer group. Similarly, when there is a situation were the person falls from the wheelchair a similar notification will be sent out stating that the patient might be in trouble (Shuo, 2015; Muhammad et al., 2017).

\section{$F$. Implemented Images}

In the above images (Fig. 8 to 11 ), there are two Arduino boards, Arduino UNO and Arduino Nano. The reason to have two is to reduce the problems of connecting multiple sensors, here the Arduino UNO and the GSM board are interlinked and connected to one Arduino Nano board resulting in only one power connection.

The main sensors are interconnected to the Android Nano Board making it as a centralized hub.

To summarize the entire implementation process, once the Arduino boards are turned $\mathrm{ON}$ and all the sensors are connected properly, the system starts functioning its basic task that is the pulse sensor will first we attached across an individual's index finger which returns that person's heartbeat and displays it in real time in the serial monitor of the Arduino IDE and at the same time the data will be visualized in the form of a graph in the Thingspeak IDE for further analysis of data. At the same time, the accelerometer sensor will be running behind which is attached to the person's wheelchair, if so there is any change in the constant value an immediate notification will be sent out to the individual's peer groups.

Similarly, when there is an emergency rising due to the overshoot or undershoot of the heart rate value over the predefined value, an alert will be sent out to the peer groups using the GSM technology.
To calculate the pulse per minute to differentiate the heart beat from one second to another the formula used was:

Pulse per Minute $=\Delta T$,

where, $T$ is the time that is time taken from second heartbeat to the first beat will be subtracted to derive the value for Pulse per Minute (T2-T1).

The constant value chosen for setting the overshoot and undershoot heart rate value is based on a detailed survey conducted by WHO, which states the prescribed heart rate ranging value for an individual will commonly range between 60-120, so these values are taken into consideration while setting the constant value for a heartbeat. If so there is an undershoot of heart rate value which is less than 60 or overshoot of heart rate value which is more than 120 an immediate alert is sent out and at the same time the data can is displayed across the Thingspeak interface were further diagnosis by the patient's health advisor can be carried out.

\section{Testing Phase}

To make sure of the efficiency for the proposed system various test cases was implemented across the different modules of the developed system.

The Figure 12 showed the provided input and expected output from various components of the proposed system. Based on the results derived all the components worked as expected and provided the desired results.

\section{Results and Discussion}

The developed system was tested among a closed group of elderly people and people who are stabilized in wheelchairs. The data was collected from a group of 24 people and later analysed. The tool used for data analysis purpose was Excel. 


\begin{tabular}{|c|c|c|}
\hline Test Case & Input & Output \\
\hline Pulse Sensor & Heart rate & $\begin{array}{c}\text { Detect an individual's } \\
\text { heart rate }\end{array}$ \\
\hline Full Detection Sensor & Force applied & $\begin{array}{c}\text { Based on the } \\
\text { speed and angle in } \\
\text { which the sensor } \\
\text { falls, values vary }\end{array}$ \\
\hline Thingspeak Module & Push Button Press & $\begin{array}{c}\text { Live heart will be shown } \\
\text { in interface from for } \\
\text { future diagnosis. }\end{array}$ \\
\hline GSM Module & 12v Input & $\begin{array}{c}\text { Latitude and longitude of } \\
\text { the current user will be } \\
\text { sent to the peer group. }\end{array}$ \\
\hline
\end{tabular}

Fig. 12: Testing reports

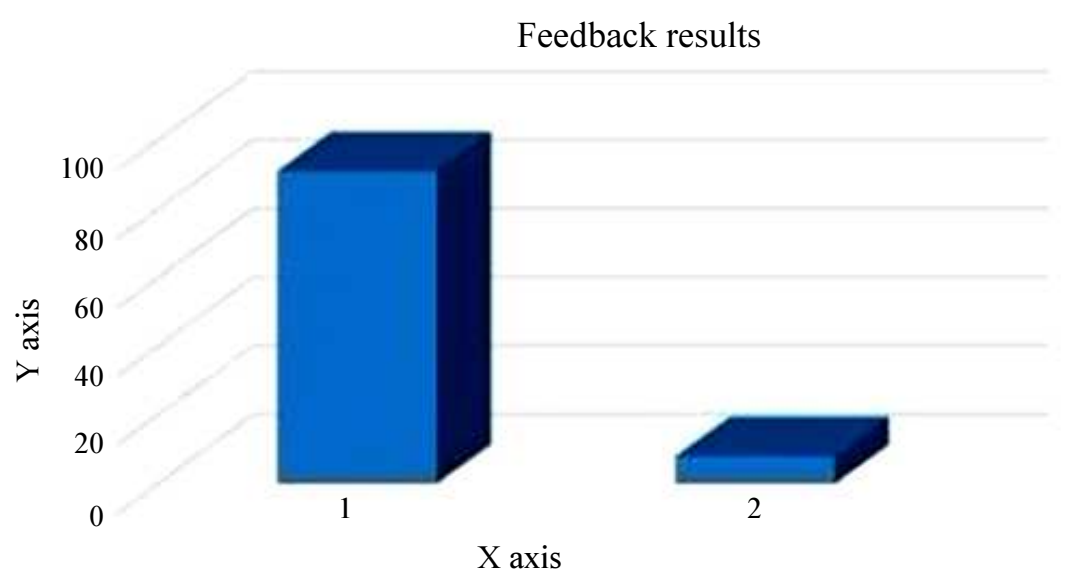

Fig. 13: Excel derived feedback results

In the above Fig. 13, there are two data points, numbered, as one and two respectively. The overall percentage considered for the derived result was 100 . The result came in favor of the proposed system were $91 \%$ of the elderly patients and people who were stabilized in wheelchair suggested that the proposed system could bring in a change in the traditional method of monitoring heart attacks and patient falls.

\section{Conclusion}

The main idea of this paper is to bring in a must need change in the traditional method of monitoring elderly people and patients who are stabilized in a wheelchair.

Even though there are various traditional systems, which do the task of monitoring heart rate, or detecting patient falls, there is no such system, which considers both these facts together. A survey states that $40 \%$ of the heart attacks are caused across the elderly population after the patient falls. So by monitoring both these rates at regular intervals, it becomes an easier task for health advisors to predict the heart attacks well in advance, by doing so necessary medications can be provided which can save a person's life before any life-threatening situation arises.

The developed system is a working prototype to remotely monitor the patients from time to time by considering the major parameters such as heart rate and fall detection, which was implemented using Arduino boards, pulse sensor and accelerometer sensor. The derived results were also made available for further diagnosis in the form of visual graphs using the Thingspeak technology. An alert or notification will also be sent out to the patient's peer groups at the time of emergency using the GSM module (Chan et al., 2013; Muhammad et al., 2017). 
The future work of this proposed system is to convert this prototype model into a full-fledged working product and make it available in the market for better usage. While considering the fact of a fullfledged product, a set of advancements available at that time of market expectations will also be incorporated within the proposed system to make it an effective product to work along (Rajalakhshmi and Nikilla, 2016; Viet et al., 2012).

\section{Acknowledgement}

This paper study was conducted in Christ University under the guidance of Prof.Monisha Singh, Prof. Joy Paulose and Dr.Rohini V as a partial requirement for the degree Master of Computer Applications.

\section{Author's Contributions}

This paper mainly focuses upon the structural build of a patient monitoring system which was guided by Ms.Monisha Sigh and built by Mr.Manikandan S under her guidance.

\section{Ethics}

The paper was derived after successful implementation of the proposed system. The system owns $100 \%$ originality and follows all the mentioned guidelines.

\section{References}

Abdelgawad, A. and K. Yelamarthi, 2016. Structural health monitoring: Internet of things application. Proceedings of the IEEE 59th International Midwest Symposium on Circuits and Systems, Oct. 16-19, Abu Dhabi, UAE, pp: 1-4.

DOI: 10.1109/MWSCAS.2016.7870118

Ahn, Y.W., A.M.K. Cheng, J. Baek, M. Jo and H.H. Chen, 2013. An auto-scaling mechanism for virtual resources to support mobile, pervasive, real-time healthcare applications in cloud computing. IEEE Net., 27: 62-68.

DOI: 10.1109/MNET.2013.6616117

Chan, A.M., N. Selvaraj, N. Ferdosi and R. Narasimhan, 2013. Wireless patch sensor for remote monitoring of heart rate, respiration, activity and falls. Proceedings of the 35th Annual International Conference of the IEEE Engineering in Medicine and Biology Society, Jul. 3-7, IEEE Xplore Press, Osaka, Japan, pp: 6115-6118.

DOI: 10.1109/EMBC.2013.6610948
Delahoz, Y.S. and M.A. Labrador, 2014. Survey on fall detection and fall prevention using wearable and external sensors. Sensors, 14: 19806-19842. DOI: $10.3390 / \mathrm{s} 141019806$

Kefyalew, E.Y. and A.R.A. Mohamed, 0000. Design and implementation of an accident fall detection system for elderly.

Li, B., Q. Gui, H.B. Ali, H. Li and Z. Jin, 2016. A wearable sit-to-stand detection system based on angle tracking and lower limb EMG. Proceedings of the IEEE Signal Processing in Medicine and Biology Symposium, Dec. 3-3, IEEE Xplore Press, Philadelphia, PA, USA, pp: 1-6. DOI: 10.1109/SPMB.2016.7846876

Muhammad, G., S.K.M.M. Rahman, A. Alelaiwi and A. Alamri, 2017. Smart health solution integrating IoT and cloud: A case study of voice pathology monitoring. IEEE Commun. Magazine, 55: 69-73. DOI: 10.1109/MCOM.2017.1600425CM

Rajalakhshmi, S. and S. Nikilla, 2016. Real time health monitoring system using arduino. South Asian J. Eng. Technol., 2: 52-60.

Satija, U., B. Ramkumar and M.S. Manikandan, 2017. Real-time signal quality-aware ECG telemetry system for IoT-based health care monitoring. IEEE Internet Things J., 4: 815-823.

DOI: $10.1109 /$ JIOT.2017.2670022

Shuo, C., 2015. Fall detection system using arduino fio.

Viet, V.Q. and D. Choi, 2012. Fall detection based on movement and smart phone technology. Proceedings of the IEEE RIVF International Conference on Computing and Communication Technologies, Research, Innovation and Vision for the Future, Feb. 27-Mar. 1, IEEE Xplore Press, Ho Chi Minh City, Vietnam, pp: 1-4. DOI: 10.1109/rivf.2012.6169847

Xie, H., X. Tao, H. Yel and J. Lu, 2013. WeCare: An intelligent badge for elderly danger detection and alert. Proceedings of the IEEE 10th International Conference on and 10th International Conference on Autonomic and Trusted Computing Ubiquitous Intelligence and Computing, Dec. 18-21, IEEE Xplore Press, Vietri sul Mere, Italy, pp: 224-231. DOI: 10.1109/UIC-ATC.2013.104 